\title{
Le mystère Mélisande : une analyse sémiostylistique du personnage de l'opéra de Debussy
}

\author{
Anne Claire GIGNOUX (Lyon)
}

\section{Summary}

Mélisande, the central figure of Debussy's opera (1902), uses verbal language as well as musical language. The present study intends to carry out a semiotic analysis of her character made of mystery and neurosis (as is usual with several female heroines in the second half of the nineteenth century). Relying on intersemiotic and stylistic devices, the analysis of various scenes in which Mélisande appears will show how Debussy's Pelléas et Mélisande manages to combine text and music.

\section{L'intersémiotique des arts}

L'objet de cette étude est l'analyse sémiostylistique, dans une perspective de sémiotique comparée des arts ou intersémiotique, du personnage de Mélisande - figure centrale de l'opéra de Debussy. Il s'agit d'appliquer les méthodes de l'intersémiotique des arts à l'étude d'une héroïne d'opéra, dont le langage est en même temps verbal et musical. Le terme d'intersémiotique apparaît d'abord chez Jakobson, et y est défini comme le rapport " d'un système de signes à un autre, par exemple de l'art du langage à la musique, à la danse, au cinéma ou à la peinture " (Jakobson 1963, 86). Par la suite ce terme a été abondamment repris et développé par Georges Molinié, notamment dans Sémiostylistique - L'effet de l'art, comme l'étude du traitement sémiotique d'un art dans la matérialité d'un autre art (cf. Molinié 1998, 41). Pour comparer deux langages artistiques différents, il est en effet nécessaire de s'appuyer sur une analyse sémiotique, qui permet de mettre en regard les moyens expressifs de chaque média artistique.

Pour revenir à Debussy, il nous a paru que les moyens verbaux et musicaux se rejoignent pour exprimer le caractère mystérieux et trouble de l'héroïne. En effet, on a souvent dit de cet opéra qu'il était plein de mystère ${ }^{1}$, d'obscurité, d'angoisse, mais cette impression est à relier certainement plus à Mélisande, personnage nuancé et trouble, qu'au lumineux Pelléas ou au cruel Golaud. Mélisande est aussi, par sa voix, entre deux mondes : celui de la mu- 
sique, et celui de la littérature - puisque, cela n'est pas toujours le cas dans l'art lyrique, le livret est, à quelques rares modifications près, une œuvre littéraire, une pièce de théâtre de l'écrivain symboliste Maurice Maeterlinck, pièce dont Mallarmé avait justement apprécié la beauté en ces termes : " [I]l semble que soit jouée une variation supérieure sur l'admirable vieux mélodrame. Silencieusement presque et abstraitement au point que dans cet art, où tout devient musique dans le sens propre, la partie d'un instrument même pensif, violon, nuirait par son inutilité. » (Mallarmé cité dans Backès 1994, 215)

L'art lyrique unit ici la musique et la littérature - et l'on connaît aussi l'importance qu'attachait Debussy au texte ${ }^{2}$, que sa musique cherche à mettre en valeur et à transposer au plus près, en utilisant une ligne de récitatif ou parlando qui exclut tout artifice lyrique et toute virtuosité inutile. ${ }^{3}$ Maurice Emmanuel, dans son ouvrage d'analyse musicale sur l'opéra de Debussy, décrit ainsi ce style de " parole parlée » : " [Debussy] donne à la cantilène une allure discrète, sans écarts mélodiques, sans secousse de rythme. " (Emmanuel 1950, 124) Ce parlando s'oppose donc à la fois à la virtuosité rythmique et mélodique de l'air, et au récitatif traditionnel de l'opéra, qui est certes plus proche de la parole, mais n'est que ponctué d'accords du clavecin ou de l'orchestre ; il s'oppose aussi au "Sprechsingen de Wagner, sous lequel court une symphonie continue » (Emmanuel 1950, 126). Il s'agit donc bien d'un tournant majeur par rapport à la musique du XIXe siècle, puisque Debussy tente d'adapter la musique à l'expression du texte et refuse de subordonner le texte à la musique. Ce style parlé, le compositeur le justifie ainsi :

À l'audition d'une œuvre, le spectateur est accoutumé à éprouver deux sortes d'émotions bien distinctes : l'émotion musicale d'une part, l'émotion du personnage de l'autre ; généralement, il les ressent successivement. J'ai essayé que ces deux émotions fussent parfaitement fondues et simultanées. (Debussy cité dans Lockspeiser/Halbreich 1980, 709)

Christian Goubault affine quelque peu la description de ce parlando qui connait, selon lui, différentes réalisations :

Nous pensons que Debussy met principalement en œuvre un type intermédiaire, un arioso qui s'oriente, selon les cas, vers le parlando ou vers un cantabile, une « cristallisation » mélodique plus ou moins longue, avec toutes les gradations que cela implique dans un sens ou dans un autre. (Goubault 2012, 197)

Par ailleurs, les critiques déplorent souvent la pauvreté des livrets d'opéra, tant dans l'intrigue que dans le texte lui-même. Debussy, habitué dans ses mélodies à travailler sa musique sur les poèmes des plus grands écrivains (Verlaine, Baudelaire ou Mallarmét, par exemple), a été inspiré par le texte de Maeterlinck, dont la pièce fut pour lui une révélation, et notamment par le personnage de Mélisande. ${ }^{5}$

Notre projet, dans cette perspective intersémiotique, est de tenter de caractériser stylistiquement (verbalement et musicalement) ce personnage et d'étudier les marques formel- 
les de son instabilité existentielle. ${ }^{6}$ Le caractère des personnages, dans Pelléas et Mélisande, est ce qui fait avancer l'action, et une grande partie du drame naît du caractère funeste de l'héroïne. ${ }^{7}$ L'analyse musicale tentera de cerner cet " énorme surcroît de signification que la musique de Debussy apporte à sa pièce [de Maeterlinck] " (Lockpeiser/Halbreich 1980, 253). Nous ne reviendrons pas sur l'éternel débat de la signification de la musique. L'intersémiotique des arts s'appuie sur une sémiotique comparée du littéraire et du musical ; la sémiotique musicale (cf. Nattiez 1975 et 1987) postule que la musique est un système de signes comparable au langage verbal, et donc par nature signifiant. Le cas de l'opéra est un peu particulier, puisqu'il représente l'idéal de l'œuvre d'art total, alliant signes auditifs (le musical et le verbal) et signes visuels (par la mise en scène). ${ }^{8}{ }^{3}$ 'est seulement la coexistence du langage musical et du langage verbal qui nous intéresse ici, et notre étude associera les remarques stylistiques sur les deux médias artistiques - puisque l'opéra est le lieu par excellence d'une analyse intersémiotique.

\section{La parole de Mélisande}

Le personnage central de l'opéra, malgré la hiérarchie du titre Pelléas et Mélisande, est bien Mélisande. C'est elle que l'on suit, depuis sa rencontre par Golaud jusqu'à sa mort. Personnalité impulsive, infantile et narcissique, elle paraît atteinte de mélancolie, rongée par la solitude, l'ennui, une angoisse diffuse et flottante. À la fin de l'œuvre, elle se comporte même de manière autodestructrice. Comment le double langage - verbal et musical - permet-il de révéler la personnalité limite de Mélisande ?

Un opéra étant une œuvre théâtrale, le personnage ne peut s'y révéler que dans son propre discours ou dans le discours que les autres tiennent sur lui. Au-delà des portraits faits par les personnages secondaires', l'opéra dispose d'un moyen privilégié pour faire connaître aux spectateurs les héros et leurs états d'âme : c'est l'aria. Or Debussy, à la suite de Wagner, compose un opéra en musique continue, renonçant à la succession de numéros indépendants, laquelle facilitait le découpage en airs ; mais il refuse aussi de laisser le lyrisme musical prendre le pas sur l'action, si bien que les chanteurs n'ont quasiment jamais l'occasion d'exprimer leur virtuosité ni leur lyrisme lors d'airs. De plus, la pièce de Maeterlinck ne contient pas de monologues, qui pourraient être à l'origine d'airs. En revanche, Maeterlinck a placé une scène où Mélisande chante à la fenêtre de la tour en se peignant les cheveux (III.1), et Debussy a conservé cette scène. ${ }^{10}$ Cet air - si on peut l'appeler ainsi - ne nous apprend pas grand chose sur Mélisande, mais participe au contraire du mystère qui l'entoure. Cette monodie en mode de ré ${ }^{11}$ non accompagnée se présente, par son texte et sa musique, comme une chanson médiévale très dépouillée. En outre, Mélisande ne parle pas d'elle, mais chante. Elle se glisse ainsi dans la peau d'un autre énonciateur, ce que l'on peut interpréter finalement comme une impossibilité de parler directement de soi.

Ce n'est donc pas dans cette chanson qu'il faut chercher l'identité de Mélisande, mais bien dans ses paroles, notamment lors de moments privilégiés où elle est confrontée aux 
hommes : sa rencontre avec Golaud (I.1) ; la scène de l'anneau avec Pelléas ${ }^{12}$ (II.1) ; le premier affrontement avec Golaud (II.2) ; l'aveu d'amour de Pelléas et Mélisande (IV.4) et enfin la mort de l'héroïne $(\mathrm{V})$.

\section{Une parole fuyante (acte I)}

La première scène est bien entendu primordiale, et le discours de Mélisande dans la forêt laisse entendre un certain nombre de marques stylistiques verbales et musicales que l'on peut mettre en parallèle.

Dans un premier temps, le caractère impulsif, émotif et instable du personnage est à relier avec des figures de répétition qui mettent en valeur son affolement ; épizeuxes, anaphores, ou épiphores, qui émaillent tout son discours :

Ne me touchez pas! ne me touchez pas! [...]

Ne me touchez pas! ne me touchez pas... [...]

Oh ! oui ! oui ! oui ! [...]

Tous! tous! [...]

Je ne veux pas le dire! je ne peux pas le dire! [...]

Je me suis enfuie! enfuie.. enfuie... [...]

Je suis perdue ! perdue ! Oh ! oh ! Perdue ici... Je ne suis pas d'ici... Je ne suis

pas née là... [...]

Oh! oh! loin d'ici... loin... loin... [...]

Je préfere mourir... mourir tout de suite ! [...]

Oui, oui... (mesures 66-113) (13 $^{13}$

Si à présent nous regardons la partition, nous constatons immédiatement que ces épizeuxes ${ }^{14}$, loin d'être des procédés musicaux artificiels de répétition et de vocalisation sur les mots, expriment simplement l'émotion du personnage, en suivant une diction très naturelle, où chaque syllabe de texte correspond à une note, et où le rythme musical épouse le rythme prosodique. On remarque alors que Mélisande, au début de la rencontre, s'exprime de préférence au moyen de doubles croches, s'opposant au rythme plus posé des triolets de Golaud. On pourra donc mettre en parallèle cette expression haletante, affolée, avec les épizeuxes.

Une autre caractéristique de Mélisande est son caractère énigmatique, qui en fait un être erratique, sans passé ni avenir, "nimb[é] de mystère " (Bosc 2011, 51), ce qui est indiqué dès cette scène d'exposition. Les critiques ont eu vite fait de présenter Mélisande comme une des femmes de Barbe-bleue et de lui assigner une identité et un passé : parce que dans Ariane et Barbe-bleue, largement postérieur (paru en 1901), une des femmes s'appelle Mélisande. Mais aucune référence n’y est faite dans notre œuvre et le rapprochement paraît aussi incertain qu' infructueux. ${ }^{15}$ 
Le dialogue entre Mélisande et Golaud est très difficile, parce que celle-ci ne peut apporter aucune réponse aux questions de Golaud ; elle lui oppose une stratégie (consciente ou inconsciente) d'évitement, observable par exemple dans l'emploi de déictiques dont rien ne permet d'identifier le référent : " perdue ici... je ne suis pas d'ici... je ne suis pas née là... " (chiffre ${ }^{16} 12$, mes. 92-93). L’usage simultané d' "ici " et «là ", normalement différenciés, accroît la confusion.

Les pronoms sont également flous, qu'il s’agisse d’indéfinis ("Tous! tous!", chiffre 10, mes. 74-75) ou de pronoms personnels anaphoriques sans référent clair ( « je ne peux pas le dire ", chiffre 10, mes. 76-77 ; "c'est la couronne qu'il m’a donnée ", chiffre 13, mes. 100101). La situation n'étant pas clairement définie, on peut même y lire une prémonition de l'aventure qui va suivre (un autre homme va combler Mélisande de cadeaux et elle voudra le fuir, mais sera obligée de mourir pour lui échapper).

Dans le même sens, les phrases se signalent parfois par leur incomplétude sémantique : " je me suis enfuie!" (chiffre 11, mes. 86) - incomplétude soulignée par la question de Golaud « oui, mais d'où vous êtes-vous enfuie » (chiffre 11, mes. 88-89) ; ou, au niveau non plus syntaxique mais discursif, par une tendance générale à éluder les questions de Golaud ; depuis la réponse évasive ("Y a-t-il longtemps que vous avez fui ? - Oui, oui... qui êtesvous ?", chiffre 15, mes. 127-129) jusqu'à l'absence de réponse ("Quel âge avez-vous ? - Je commence à avoir froid ", chiffre 18, mes. 152-154). Ainsi, les questions fondamentales sur l'essence de Mélisande ne trouvent-elles aucune réponse.

Cette stratégie pourrait être celle de quelqu'un qui ne souhaite pas être interrogé, mais c'est, plus profondément, le signe d'un personnage en souffrance dans son être, car les autres dialogues de l'opéra vont confirmer cette attitude de Mélisande, même quand elle n'est pas apeurée, même quand elle est face à l'homme qu'elle aime.

Ces réponses sibyllines sont parfaitement soutenues par la ligne mélodique, marquée par un flou harmonique et une absence de cadence qui perdent peu à peu la tonalité ; on a pu parler de "suspension harmonique ». Le jeu de silence, caractéristique remarquable de Debussy, et la finesse de la dynamique y concourent aussi. On note, par exemple, que les deux premières répliques de Mélisande (chiffres 7 et 8, mes. 63 et 66-67) ont lieu dans un silence absolu de l'orchestre (en contraste avec les phrases de Golaud qui les entourent), tandis que la troisième (" oh oui ", chiffre 10, mes. 75) n'est soutenue que par deux accords de cordes. La suivante est marquée par une grande agitation des cordes, tandis que l'information indicible que Mélisande ne peut pas délivrer semble suggérée par la phrase mélancolique des hautbois, qui suppléent à son mutisme. Dans la réplique suivante (« je me suis enfuie », chiffre 11, mes. 86-87), la fuite de Mélisande symbolisée par la montée jusqu’au sol aigu de la mélodie, est accompagnée de rares pizzicati aux cordes, évoquant la disparition de la jeune femme (mais aussi son errance existentielle). On notera encore la réplique suivante, « je suis perdue » (chiffre 12, mes. 90-92), marquée par un chromatisme descendant à la basse et aux violons, et un rythme effréné de quintes augmentées aux vents, qui en ‘ noyant le ton disent cet égarement de Mélisande. Enfin, les répliques de Mélisande sont chantées piano, les instruments qui l'accompagnent jouent piano voire pianissimo, signe d'une parole qui n'ose s'affirmer. 
Le dernier point stylistique que nous voudrions interroger dans cette scène est celui de la violence contenue en Mélisande qui, là encore, trouve une expression verbale et musicale à la fois. C'est la dynamique qui met l'accent sur cette violence retenue : deux phrases sont en effet prononcées dans la nuance forte. Dans la première, «non! non! je n'en veux plus! je n'en veux plus! je préfere mourir " (chiffre 14, mes. 107-111), un crescendo conduit jusqu’à " je préfere mourir ", asséné en noires, et soutenu par un tutti de l'orchestre. L'autre phrase (ce sont les deux seules forte de toute la scène) est "si vous la retirez, je me jette à sa place! " (chiffre 14, mes. 119-121), et fonctionne selon le même principe : rythme plus lent, plus martelé (noires au lieu des croches ou doubles croches habituelles), crescendo, forte de tout l'orchestre. Or dans ces deux phrases ${ }^{17}$, Mélisande évoque sa mort à venir ; non seulement par une prescience propre aux personnages tragiques (" je préfere mourir tout de suite »), mais aussi parce que la mort lui est familière, le suicide peut lui apparaittre comme une solution impulsive et réaliste face à sa solitude.

\section{Les sentiments suggérés par la musique (acte II)}

Un peu plus loin dans l'œuvre (II.2), Mélisande se trouve à nouveau confrontée à Golaud, maintenant son époux depuis plus de six mois, alors que sa vie dans ce château lugubre et son attirance naissante pour Pelléas la rendent encore plus vulnérable qu’avant. Elle apparaît comme un être qui n'existe presque $\operatorname{pas}^{18}$, pris entre deux mondes, celui de la réalité et celui de ses rêves, sur une frontière inconfortable dont elle ne comprend pas les signes.

Dans la deuxième partie de cette scène, elle se met à sangloter, et son mari l'interroge sur les raisons de sa tristesse. Les réponses qu'elle lui fait sont pour la plupart de polarité négative, soit qu'elle nie les hypothèses de son époux : « je ne suis pas heureuse ici » (chiffre 19, mes. 225-226), " personne ne m'a fait le moindre mal... ce n'est pas cela " (chiffre 19, mes. 229-230), « ce n'est pas Pelléas. Ce n'est personne...» (chiffre 20, mes. 237-238), « ce n’est pas cela " (chiffre 21, mes. 246-247), "c'est ici que je ne peux plus vivre " (chiffre 21, mes. 249-250), « ce n'est pas cela, ce n'est pas cela " (chiffre 24, mes. 269); soit qu'elle nie à son époux toute possibilité de la comprendre, dans son absolue solitude : "Vous ne pouvez pas me comprendre » (chiffre 20, mes. 239) ; soit enfin que, sur le mode épistémique, elle avoue son incapacité à se comprendre elle-même : "Je ne sais pas " (chiffre 19, mes. 222). Ces mots ne sont pas à interpréter comme une dérobade de femme adultère, mais comme le sincère aveu d'une impuissance fondamentale à lire en soi ${ }^{19}$; elle parle de "maladie " (chiffre 19, mes. 219), mais cette maladie n'est visiblement pas physique. On note donc dans son discours la répétition de phrases négatives, centrées à nouveau autour de deux déictiques prédicatifs : " cela " et « ici ». Le " cela " vise à déterminer la souffrance en question, mais Mélisande ne parvient pas à l'approcher. Le « ici » est un complément circonstanciel de lieu nettement prédicatif : « je suis malade ici » (chiffre 19, mes. 219-220 et 223), « je ne suis pas heureuse ici » (chiffre 19, mes. 225-226), "c'est ici que je ne peux plus vivre " (chiffre 21, mes. 249250). L'origine de ce malaise persistant semble donc tenir au lieu même, le château se révélant 
finalement, comme la forêt, un endroit impossible à vivre pour Mélisande - comme aussi l'endroit qu'elle a dû fuir au début de la pièce. Il lui est impossible de se sentir bien où que ce soit. La mort, à nouveau évoquée, semble être la seule solution : "c'est ici que je ne peux plus vivre... je sens que je ne vivrai plus longtemps » (chiffre 21, mes. 249-251). La disparition du " ici » favorise un nouveau sens de "vivre», pris (par antanaclase) non plus dans son sens de 〈 habiter `, mais dans son sens premier ` exister `. L'être utopique ne peut plus rester sur terre.

Du point de vue de l'orchestration, un contraste subtil oppose Golaud à Mélisande. On sait que l'orchestration de Pelléas et Mélisande est particulièrement travaillée, et que les doublures aussi bien que les tutti y sont rares, Debussy leur préférant les instruments solistes et la couleur orchestrale. Or, la plupart du temps, Golaud est accompagné par un nombre plutôt important d'instruments : les cordes, les bois (clarinettes, hautbois, bassons) et aussi, de façon très caractéristique, les cors, qui disent en même temps sa virilité et son caractère de chasseur. Certes, dans cette scène de discussion intime entre un mari et sa femme, Golaud, à plusieurs reprises, peut-être même influencé par la douce évanescence de Mélisande, se prend à chanter a cappella. Mais en observant la partition, on voit que Mélisande est accompagnée soit par quelques cordes (par ex, sans le violoncelle ${ }^{20}$; ou au contraire, sans les violons et $\left.\mathrm{alti}^{21}\right)$; soit par les violons et deux flûtes uniquement ${ }^{22}$; soit par des tenues ou des pizzicati aux cordes ${ }^{23}$. Parfois l'orchestre devient un peu plus animé ("Oh non, ce n’est pas cela, je voudrais m’en aller avec vous ", chiffre 21, mes. 246-249) et Mélisande plus véhémente, mais cela reste très fugitif : elle finit sa phrase dans un pianissimo ("retenu ») et dans le médium grave de sa tessiture, évoquant sa mort comme en un souffle ( je sens que je ne vivrai plus longtemps ", chiffre 21, mes. 250-251).

Il existe cependant une échappatoire à Mélisande, que seule la musique suggère : il s'agit de l'amour qui naît en elle pour Pelléas, qui crée pour elle un véritable lieu où vivre, où s'accomplir. Au moment où Golaud lui parle de Pelléas, Mélisande, entre son texte et la musique, se contredit. Ce qu'elle énonce : "il ne m'aime pas, je crois » (chiffre 22, mes. 259-263), relève d'une verbalisation consciente de son inquiétude existentielle, de sa carence affective qui l'empêchent de comprendre clairement que Pelléas est amoureux d'elle. Mais, dans la partition, déjà dans les répliques précédentes, Debussy a commencé à faire apparaître le thème de Pelléas avec sa quarte caractéristique : sous forme de quartes diminuées au cor anglais ${ }^{24}$, puis de quartes justes au violoncelle juste après (chiffre 20, mes. 233). Enfin lorsque Mélisande dit ne pas savoir d'où vient sa souffrance ("c'est quelque chose qui est plus fort que moi ", chiffre 20, mes. 240-241), la flûte fait à nouveau entendre, plus clairement cette fois, le thème de Pelléas, évoquant donc cette passion de Mélisande pour Pelléas. Quand Mélisande ajoute "il ne m’aime pas, je crois " (chiffre 22, mes. 260-261), c'est le hautbois qui joue cette fois ce thème, et quand elle finit " mais il me parle quand il me rencontre " (chiffre 23, mes. 264-265), les premiers violons font entendre un petit motif alerte 25 qui est une citation de la scène de l'anneau (au chiffre 9 de l'acte II, mes. 73-74), la scène où ils se sont parlé et où Mélisande $\mathrm{a}$, par un acte manqué, perdu son anneau de mariage en présence de Pelléas. Mélisande, personnage errant, utopique, aurait ainsi trouvé un lieu, un ailleurs où vivre enfin heureuse et réunie : l'amour. Cela, seule la musique nous l'annonce ici, 
par le jeu des citations de motifs musicaux. Ainsi, Debussy a réalisé l'œuvre dont il rêvait : " la musique y commence là où la parole est impuissante à exprimer : ‘ la musique est faite pour l'inexprimable ${ }^{2} »^{26}$

\section{La révélation lyrique (acte IV)}

Bien sûr, un peu plus tard, cet amour se révèle au grand jour (IV. $\left.4^{27}\right)$. Il s'agit également, pour l'héroïne, d'une scène de révélation, de bouleversement, où dans une pulsion de mort impulsion, elle décide subitement de mourir pour échapper à son inexistence et sublimer l'amour dans la mort. Cette impulsion va se signaler, tant dans le texte que dans la musique, par la présence d'un lyrisme auquel le parlando et la retenue du reste de la partition ne nous avaient pas habitués. La révélation de Mélisande à elle-même se fait cependant de manière progressive ; d'abord chuchotant, son discours se fait plus affirmatif : on ne retrouve plus les épizeuxes si caractéristiques de sa difficulté à exister, elle souhaite au contraire exister au grand jour : " laissez-moi dans la clarté " (chiffre 38, mes. 572), " je veux qu'on me voie " (chiffre 38, mes. 575-576), deux fois « oui » (chiffre 38, mes. 577 et chiffre 37, mes. 568), «j'ai couru " (chiffre 39, mes. 585) : par leur modalité assertive et leur efficacité pragmatique (qui vient de leur simplicité syntaxique), ces phrases témoignent d'une nouvelle assurance chez Mélisande. Cette nouvelle assurance est remarquée par Pelléas (" qu'as-tu donc ? " chiffre 38, mes. 576) qui la compare encore malgré tout à un « oiseau pourchassé » (chiffre 39, mes. 587588) - l'oiseau étant la métaphore récurrente de Mélisande. L'instant même de la déclaration d'amour est un moment grave et secret, proféré dans le silence total de l'orchestre, chanté par Mélisande dans son registre grave, et toujours sur un style parlando (chiffre 42, mes. 618619). Puis un élan lyrique incontrôlé emporte Pelléas dans son air en fa dièse (à partir du chiffre 44), et la force de cet élan amoureux semble se communiquer à Mélisande ; mais en réalité, celle-ci ne devient lyrique à son tour qu'à partir du moment où la mort s'approche (lorsque les portes du château sont fermées, et que l'ombre de Golaud apparaît). Dans un premier temps, juste après la déclaration d'amour, Mélisande reste l'être de clair-obscur qu'elle a toujours été : " je suis plus près de toi dans l'obscurité » (chiffre 49, mes. 680-681), « je te voyais ailleurs " (chiffre 49, mes. 687), " si, si, je suis heureuse, mais je suis triste » (chiffre 49, mes. 689-691). Elle continue de s'exprimer dans un relatif silence de l'orchestre, et dans la nuance piano; les quelques instruments qui l'accompagnent, comme les cordes sur " je te voyais ailleurs » et le cor anglais et la clarinette sur « je suis heureuse mais je suis triste " sont d'après les indications de Debussy sur la partition " doux et expressifs "; le cor anglais rejoue d'ailleurs le thème de Mélisande, le rappel de son éternelle mélancolie. Puis l'orchestre se fait nettement expressif, avec des roulements de timbales et le retour du thème de Golaud (le leitmotiv le plus reconnaissable, grâce à son rythme pointé, chiffres 49-50). Et tandis que l'orchestre s'anime, les deux héros s'exaltent, et Mélisande, une première fois, affirme son désespoir de vivre à Pelléas qui lui dit : « il est trop tard " (chiffre 50, mes. 707-709), elle répète « tant mieux ! tant mieux ! " (chiffre 50, mes. 710-712). Pelléas est étonné de cette impulsion de Mélisande, si bien qu'il 
lui répond par une aposiopèse : «tu ?... voilà, voilà !» (chiffre 50, p. 712-714). Un dernier moment décisif a lieu avec la découverte de la présence de Golaud qui les épie (chiffre 54); après un instant de frayeur, Mélisande refuse la fuite, et quand Pelléas dit «il nous tuera " (chiffre 55, mes. 796-797), elle reprend les mêmes mots qu'auparavant : « tant mieux ! tant mieux!» (chiffre 55, mes. 798-801), mais change son intervalle de tierce mineure en tierce majeure, plus lumineuse, plus affirmée. L'épizeuxe n’a plus la même valeur que celle que nous lui avions trouvée dans la toute première scène : il s'agit maintenant, au contraire, d'une affirmation, d'un défi envers la mort. Dans ce délire de toute-puissance, Mélisande et Pelléas s'embrassent passionnément sous les yeux du jaloux et trouvent l'accord lyrique des amoureux d'opéra, l'harmonie parfaite (" toutes les étoiles tombent ! - Sur moi aussi ! sur moi aussi !", chiffre 56, mes. 813-816) marquée par la symétrie de rythme (trois fois « donne! ", trois fois " toute! ", chiffre 57, mes. 819-824), laquelle s'achève sur un unisson, avant que Golaud ne porte le coup fatal à Pelléas. L’orchestre, « très animé jusqu’à la fin » (chiffre 56, mes. 812), est exceptionnellement présent. Après la mort de Pelléas, Mélisande redevient un oiseau effrayé, prisonnier de ses propres émotions, et on peut imaginer qu'elle s'évanouit. Maeterlinck dans sa pièce faisait expliquer au début de l'acte $\mathrm{V}$ par des servantes que Golaud avait poignardé Mélisande sous le sein gauche ; cette scène ayant disparu, la mort de Mélisande paraît chez Debussy sans cause explicite, plutôt comme une conséquence de son état d'inexistence, en tant qu'être toujours à la frontière de la vie et de la mort.

Une fois Pelléas mort, une fois passée l'illusion d'un lieu d'existence, qu'aurait été leur amour partagé, Mélisande redevient, pour le dernier acte, un être inexistant et mélancolique ; comme le dit Michel Bosc (2011, 135), " Mélisande, en cet instant, se trouve ramenée à sa condition errante et sans attache". La révélation a échoué ; il ne lui reste plus qu’à se laisser mourir sous les yeux de ceux qui ne la comprennent pas, au terme d'une vie qui n'a jamais eu de sens. "Oui, je n'ai plus toutes ces inquiétudes ", dit-elle avant de mourir ; le déterminant indéfini pluriel et le déictique < ces > laissent à cette instabilité existentielle son incompréhension fondamentale.

\section{Pour conclure}

L'héroïne d'opéra, dans la seconde moitié du XIXe, période où la psychanalyse s'intéresse au cas de la femme, offre souvent une cristallisation de névroses féminines. La Traviata (Verdi 1853), au surnom évocateur, n'est pas seulement une femme dévoyée; c'est aussi un être qui se perd dans l'excès et la jouissance plutôt que d'affronter son angoisse. La scène la plus célèbre de Lucia de Lammermoor (Donizetti 1835) est la scène "de la folie ", où Lucia après avoir tué son fiancé perd la raison. On peut encore penser à Madame Butterfly (Puccini 1904), murée dans un fantasme de mariage dont tout le monde sait qu'il n'existe pas, qui finit par mettre fin à ses jours. Le mystère féminin, la névrose féminine semblent être des sujets de prédilection pour l'opéra au XIXe siècle et au début du XXe siècle (que l'on pense aussi à Erwartung de Schönberg), qui permettent l'expression dramatique des mystères de 
l'inconscient - ce que la musique peut signifier de façon très efficace, comme nous l'avons montré avec le cas de Mélisande. Mélisande se révèle être "l'enjeu et le mobile du drame, tour à tour innocente puis fatale, femme paysage qui insuffle son ambiguïté aux éléments " (Daniel Risacher 2011, 54), elle demeure, comme le fait remarquer Julia Kristeva (2006, 32), le personnage central de l'opéra :

Ainsi, Mélisande cristallise-t-elle les peines et les attraits de la femme mélancolique, et, au-delà, nous confronte à cette expérience du rien, du nonêtre, que les époques progressistes ignorent ou écartent, et que les périodes de crise cultivent.

Si Mélisande n'est pas la seule héroïne emblématique de ce "sentiment d'inconnaissable qui baigne la pièce comme la fin du siècle " (Kristeva 2006, 32), l'opéra de Debussy reste cependant un modèle exemplaire dans ce domaine, en même temps qu'une réussite exceptionnelle dans l'alliance du texte et de la musique. Grâce à celle-ci, Mélisande devient celle "qui se dérobe à jamais - insaisissable et essentiellement Autre - se situant de ce fait du côté du mythe de la féminité même » (Gonnard 2015, 363).

\section{Notes}

1 Il s'agit là d'une impression partagée par de nombreux spectateurs de l'œuvre comme Jankelevitch 1977, et que l'on retrouve notamment chez Daniel-Risacher 2001 et chez Kristeva 2006, pour qui ce mystère est lié au féminin.

2 Jensen 2014, 187, souligne la réussite exceptionnelle de Debussy dans ce domaine : "[E]ven today Debussy's handling of the text seems a marvel, a synthesis of word and music that appears completely natural. But what makes it particularly effective is the role of the orchestra. Voice and orchestra are not independent. »

3 Gonnard 2015, 357, décrit avec précision la déclamation dans Pelléas et Mélisande: "On observe plus exactement une oscillation entre trois principales mises en œuvre du texte tout au long de la partition : le quasi parlando, que l'on a pu rattacher au chant grégorien comme au stile rappresentativo de Monteverdi ; un arioso ductile, à mi-chemin entre l'air et le récitatif ; enfin, une déclamation lyrique correspondant à des moments fugitifs de véritable cristallisation mélodique. »

4 Respectivement pour les Ariettes oubliées (1885-1887), les Cinq poèmes de Baudelaire (1887-1889) et les Trois poèmes de Stéphane Mallarmé(1913).

5 Michel Bosc 2011, 163, compositeur, souligne d'ailleurs la difficulté pour les chanteurs de servir l'œuvre de Maeterlinck et Debussy : "La véritable difficulté pour le chanteur, dans Pelléas et Mélisande, réside dans le fait qu'il est en devoir d'assumer pleinement le texte et de le servir autant que la musique. Cette exigence n'est pas aussi courante que l'on croit. » Par ailleurs, il est intéressant également de noter que le symbolisme littéraire, dans son projet même, porte en lui ce goût du mystère qui séduisit tant Debussy : les symbolistes, notamment belges, "valorisent évidemment 
le symbole, mais aussi l'ésotérisme, le mystère, la suggestion " (Aron/Saint-Jacques/Viala 2002, 600). L'enjeu serait donc également d'évaluer comment l'esthétique symboliste s'exprime ici aussi bien dans le texte que dans la musique.

6 Partition de référence : Pelléas et Mélisande, Durand \& Cie, 1979.

7 Jensen 2014, 188, note l'importance des caractères dans cet opéra : «Much of the drama in Pelléas occurs not in exterior action but as character development - as a response to what has become an increasingly untenable situation. "

8 Nous n'étudions pas ici de mise en scène, car elle relève d'un participant extérieur à la composition musicale : le metteur en scène. Celui-ci apporte sa vision tout à fait personnelle de l'œuvre, comme le fit par exemple Olivier Py en 2007 au théâtre Stanislavski de Moscou (cf. Gil 2013).

9 Ils sont dans cette œuvre quasi inexistants : cf. la conclusion d'Arkel à l'acte V, ou la lettre de Golaud lue par Geneviève (I.2).

10 Angelet ("Pelléas et Mélisande: des brouillons de Maeterlinck au livret de Debussy », in : Angelet 1994, 56) rappelle que cette chanson se trouvait dans la première version de Maeterlinck, avant qu'il ne lui préfere la chanson des " trois sœurs aveugles".

11 «Cela se chante sur le vieux mode de ré (en ton de mi). " (Emmanuel 1950, 91)

12 Elle a été déjà largement commentée et nous ne nous en sommes finalement pas servie dans les limites de cette étude.

13 Debussy respecte globalement le texte de Maeterlinck, sur lequel il opère cependant de légères modifications, visibles lorsque l'on compare l'œuvre originale et la partition. Nous ne nous intéressons ici qu’au texte réécrit par Debussy.

14 Elles ne proviennent pas toutes du texte original de Maeterlinck : Debussy a créé des épizeuxes, ou bien a augmenté le nombre des réduplications de deux à trois.

15 Dans Ariane et Barbe-bleue, Maeterlinck réutilise en effet les noms de plusieurs de ses héroïnes d'autres œuvres, dont Mélisande ; mais celle-ci refuse de quitter Barbe-bleue à la fin de la pièce. Cf. Maeterlinck 1979.

16 Dans la partition ‘ officielle > que j'utilise, les numéros de mesure ne sont pas indiqués, je les ai rajoutés à la main ; en revanche, ce que l'on appelle les ‘ chiffres > - c'est-à-dire les chiffres mis audessus des lignes à l'intérieur de carrés, et qui correspondent à des phrases musicales de longueur plus ou moins variable - sont de Debussy ; ils sont plus fiables que les numéros de mesure, même si on ne les voit pas toujours dans les extraits photocopiés de la partition.

17 Qui sont, pour Emmanuel 1950, 128, "chantées » et non plus simplement " parlées ».

18 "Mélisande est presque inexistante », dit Jankelevitch 1977, 136.

19 Ce qu'elle redira magistralement à l'acte $V$ : « Je ne comprends pas non plus tout ce que je dis, voyezvous... Je ne sais pas ce que je dis... Je ne sais pas ce que je sais... Je ne dis plus ce que je veux... »

20 "Je suis malade", chiffre 19, mes. 219-220.

21 "Je ne sais pas. Je suis malade ici ", chiffre 19, mes. 222-223.

22 "Je ne suis pas heureuse ici ", chiffre 19, mes. 225-226.

23 "Non non, personne ne m'a fait du mal ", chiffre 19, mes. 228-230.

24 Chiffre 20, mes. 232-234: "Mais tu dois me cacher quelque chose ", dit Golaud, tandis que le cor anglais évoque cet amour caché... 
24 Chiffre 20, mes. 232-234 : "Mais tu dois me cacher quelque chose », dit Golaud, tandis que le cor anglais évoque cet amour caché...

25 Condé dans son " Guide d'écoute » $(2012,26)$ nomme ce motif «l'injonction tendre ».

26 Debussy cité dans Kristeva 2006, 43.

27 Pour une analyse détaillée de cette scène centrale, cf. Rolf 2007, 117-148. Pour une analyse détaillée de cette scène centrale, cf. Rolf 2007, 117-148.

\section{Bibliographie}

Angelet, Christian (éd.) : Pelléas et Mélisande. Actes du colloque international de Gand. Gand : Fondation Maurice Maeterlinck, 1994.

Aron, Paul / Saint-Jacques, Denis / Viala, Alain (éds) : Le Dictionnaire du littéraire. Paris : PUF, 2002.

Backès, Jean-Louis : Musique et littérature. Paris : PUF, 1994.

Bosc, Michel : Symbolisme et dramaturgie de Maeterlinck dans Pelléas et Mélisande. Paris: L'Harmattan, 2011.

Branger, Jean-Christophe / Douche, Sylvie / Herlin, Denis (éds.) : Pelléas et Mélisande cent ans après : études et documents. Lyon : Symétrie, 2012.

Condé, Gérard : «Guide d'écoute ». In : L’Avant-Scène Opéra 266 (2012), 7-63.

Daniel-Risacher, Nathalie : "Pelléas et Mélisande, ou le désespoir de la dernière heure ». In : MartinLau, Philippe (éd.) : Centenaires de Pelléas : de Maeterlinck à Debussy. Orléans : Paradigme, 2001, 53-66.

Debussy, Claude : «Ariettes oubliées » (1885-1887). «Cinq poèmes de Baudelaire » (1887-1889). In : Debussy, Claude : Songs. Partitions. New York : Dover publications, 1981.

Debussy, Claude : Monsieur Croche et autres écrits. Paris : Gallimard, 1987.

Debussy, Claude : Pelléas et Mélisande (= L'Avant-scène. Opéra, 266). Paris : Éditions Premières Loges 2012.

Debussy, Claude : Pelléas et Mélisande. Partitions. Paris : Durand \& Cie, 1979.

Debussy, Claude : Trois poèmes de Stéphane Mallarmé. Partitions. Paris : Durand \& Cie, 1913.

Emmanuel, Maurice : Pelléas et Mélisande de Debussy. Paris : Éditions Mellottée, 1950.

Gil, Marie : "Pelléas et Mélisande, Debussy». In : L'Avant-Scène Opéra 275 (2013) (Opéra et mise en scène: Olivier Py. Paris : Éditions Premières Loges), 49-51.

Gonnard, Henri : "La chanson de Mélisande dans Pelléas (1902) de Debussy - de la technique à l'herméneutique ». In : Hascher, Xavier / Ayari, Mondher / Bardez, Jean-Michel (éds.) : L'Analyse musicale aujourd'hui. Sampzon : Éditions Delatour France, 2015, 357-365.

Goubault, Christian : "Le geste compositionnel debussyste ». In : Branger, Jean-Christophe / Douche, Sylvie / Herlin, Denis (éds.) : Pelléas et Mélisande cent ans après : études et documents. Lyon : Symétrie, 2012, 195-213.

Jakobson, Roman : Essais de linguistique générale. Paris : Minuit, 1963.

Jankelevitch, Vladimir : "Débussy, Pelléas et Mélisande». In : L'Avant-Scène Opéra 9 (1977), 131-145.

Jensen, Eric Frederick : Debussy. New York : Oxford University Press, 2014. 
Kristeva, Julia : "Pelléas et Mélisande: une mélancolie sonore ». In : Opéra de Paris (éd.) : Lectures d'opéra. Paris : Christian Bourgois, 2006, 25-47.

Lockspeiser, Edward / Halbreich, Harry : Claude Debussy. Paris : Fayard, 1980.

Maeterlinck, Maurice : Théatre complet. Genève : Slatkine reprints, 1979.

Molinié, Georges : Sémiostylistique. L'effet de l'art. Paris : PUF, 1998.

Nattiez, Jean-Jacques : Fondements d'une sémiologie de la musique. Paris : U.G.E., 1975.

Nattiez, Jean-Jacques : Musicologie générale et sémiologie. Paris : Christian Bourgois, 1987.

Rolf, Marie : "Symbolism as Compositional Agent in Act IV, scene 4 of Debussy's Pelléas et Mélisande». In : Kelly, Barbara L. / Murphy, Kerry (éds.) : Berlioz and Debussy : Sources, Contexts, and Legacies. Essays in Honour of François Lesure. Aldershot : Ashgate, 2007, 117-148. 